\title{
Multimodal evaluation of the cerebrovascular reserve in Neurofibromatosis type 1 patients with Moyamoya syndrome
}

\author{
Alessandra D'Amico ${ }^{1}$ • Lorenzo Ugga $^{1}$ - Sirio Cocozza ${ }^{1}$ (D) Sara Maria delle Acque Giorgio ${ }^{1}$ - Domenico Cicala ${ }^{2}$.

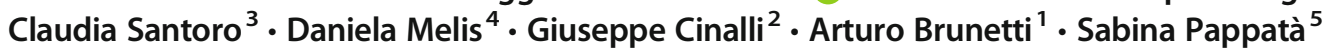

Received: 24 March 2020 / Accepted: 2 July 2020 / Published online: 10 July 2020

(C) The Author(s) 2020

\begin{abstract}
Purpose Moyamoya syndrome (MMS) is a rare intracranial arterial vasculopathy which can occur in neurofibromatosis type 1 (NF1) disease, representing a cause of cerebrovascular reserve (CVR) impairment, possibly leading to ischemic stroke. Here, we evaluated noninvasive imaging techniques used to assess CVR in MMS patients, describing clinical and imaging findings in patients affected by MMS-NF1.

Methods Following strict inclusion and exclusion criteria, in this retrospective observational study, we evaluated imaging data of nine consecutive MMS-NF1 patients $(\mathrm{M} / \mathrm{F}=5 / 4$, mean age: $12.6 \pm 4.0)$. Subjects underwent a multimodal evaluation of cerebral vascular status, including intracranial arterial MR Angiography (MRA), MRI perfusion with dynamic susceptibility contrast (DSC) technique, and 99mTc-hexamethylpropyleneamine oxime (HMPAO) SPECT.

Results In 8 out 9 patients ( $88.8 \%, 6 / 8$ symptomatic), time-to-peak maps were correlated with the involved cerebral hemisphere, while in 6 out 9 patients $(66.6 \%, 5 / 6$ symptomatic), mean transit time (MTT) maps showed correspondence with the affected cerebrovascular territories. Cerebral blood flow (CBF) calculated using DSC perfusion failed to detect the hypoperfused regions instead identified by SPECT-CBF in all patients, while MTT maps overlapped with SPECT-CBF data in all cases and time-topeak maps in $60.0 \%$.

Conclusions Although SPECT imaging still represents the gold standard for CBF assessment, our results suggest that data obtained using DSC perfusion technique, and in particular MTT maps, might be a very useful and noninvasive tool for evaluating hemodynamic status in MMS-NF1 patients.
\end{abstract}

Keywords Moyamoya $\cdot$ MRI $\cdot$ SPECT $\cdot$ Neurofibromatosis type 1

Electronic supplementary material The online version of this article (https://doi.org/10.1007/s10072-020-04574-4) contains supplementary material, which is available to authorized users.

Sirio Cocozza

sirio.cocozza@unina.it

1 Department of Advanced Biomedical Sciences, University of Naples "Federico II", Via Pansini, 5, 80131 Naples, Italy

2 Department of Pediatric Neurosurgery, Santobono-Pausilipon Children's Hospital, Naples, Italy

3 Referral Centre of Neurofibromatosis, Department of Woman and Child, Specialistic and General Surgery, University "Luigi Vanvitelli”, Naples, Italy

4 Department of Translational Medical Sciences, Section of Pediatrics, University of Naples "Federico II", Naples, Italy

5 Institute of Biostructure and Bioimaging, National Research Council, Naples, Italy

\section{Introduction}

Neurofibromatosis type 1 (NF1) is a multisystem autosomal dominant disorder caused by mutations in the neurofibromin tumor suppressor gene, mainly affecting eyes, skin, bones, and central nervous system (CNS) [1,2]. Cerebral arterial involvement is a well-recognized feature of this condition, mostly related to vessel stenosis $(2.5-6 \%$ of cases) [3, 4], although less frequent manifestations such as aneurysms or artero-venous malformations and fistulas could be present in this disease [2]. Indeed, the most frequent expression of vascular involvement in NF1 patients is a progressive and significant arteriopathy similar to those observed in moyamoya (MM) disease (MMD), regarded as MM syndrome (MMS) [5]. These conditions share a similar diagnostic workflow, clinical presentation, and outcome after surgical revascularization [6], with MMS being defined when MM occurs in 
association with a well-recognized condition (such as NF1), while subjects without known associated risk factors are classified as MMD patients [5]. Nevertheless, both conditions are characterized by a progressive intimal proliferation resulting in luminal obstruction, mostly affecting the supraclinoid internal carotid arteries (ICAs) and the proximal segment of both anterior and middle cerebral arteries (ACA, MCA). Similarly to what described for MMD, in MMS, the development of tortuous leptomeningeal collateral networks and compensatory dilations of perforating arteries produces the typical angiographic image of "puff of smoke" [7-9]. Despite digital subtraction angiography (DSA) is still considered the gold standard for diagnosis and presurgical evaluation of both MMD and MMS, MR angiography (MRA) represents a valuable diagnostic tool in these conditions [10]. Indeed, according to the "Research Committee for the Diagnosis of MMD in Japan" guidelines [10], cerebral DSA is not mandatory if MRA demonstrates ICA or proximal ACA/MCA stenosis. Moreover, abnormal vascular network with flow voids in the basal ganglia and, especially, in the Sylvian fissures on T2weighted images strengthens the diagnosis of MMD and MMS [11].

Although different grades of hemodynamic insufficiency occur in these conditions, clinical symptoms might or might not be present when diagnosis is reached [12]. Usually, the initial manifestations are related to the occurrence of ischemic events, often multiple and recurrent due to the development of the steno-occlusive lesions hallmark of this condition [13]. Transient ischemic attacks have also been reported, especially in pediatric population [14], while hemorrhagic events are more common in adults [15]. Along with symptoms related to the occurrence of ischemic events, patients can also present with headache, which has been reported to be a common clinical finding of this condition [16] that may improve after a successful revascularization surgery [17]. For these reasons and to avoid serious and invalidating complications, it is important to immediately recognize MMS. In this light, both single-photon emission computed tomography (SPECT) with acetazolamide challenge and positron emission tomography (PET) examinations represent valuable tools to assess cerebrovascular reserve (CVR) and hemodynamic impairment in MM patients, providing quantitative measures of different cerebral perfusion variables $[18,19]$. These include the relative cerebral blood flow and volume (rCBF and $\mathrm{rCBV}$, respectively), the oxygen extraction fraction (OEF) and the regional cerebral metabolic rate for oxygen $\left(\mathrm{rCMRO}_{2}\right)$, all parameters that help in selecting patients at higher risk of stroke and therefore requiring a revascularization surgery, given their association with severe hypoperfusion and marked hemodynamic failure [20].

Nevertheless, these imaging procedures are known to be relatively invasive, exposing young patients to ionizing radiation. For this reason, in recent years, less invasive perfusion techniques using MRI have been proposed, such as arterial spin labeling (ASL), dynamic susceptibility contrast perfusion weighted imaging (DSC-PWI), or $\mathrm{CO}_{2}$-triggered bloodoxygen-level-dependent (BOLD) functional MRI, reported to have a similar effectiveness to evaluate CVR in both MMD and MMS patients [21, 22]. Indeed, it has been recently reported that MR-derived perfusion parameters (namely, the mean transit time (MTT)) negatively correlated with CVR measured with SPECT and acetazolamide challenge in MMD patients, suggesting that DSC-MRI may provide valuable information about the CVR in these patients [21].

Given this background, the aim of the study was to expand the current knowledge about the evaluation of CVR in MMSNF1 patients, by (i) describing clinical and imaging findings in subjects undergoing a multimodal imaging evaluation; (ii) investigating a possible role of noninvasive imaging techniques, such as DSC-PWI, in the evaluation of CVR in MMS-NF1 patients; and (iii) comparing SPECT and MRI derived cerebral perfusion parameters in a subgroup of patients.

\section{Material and methods}

\section{Participants}

In this retrospective observational study, we reviewed data of NF1 patients clinically evaluated between January 2007 and December 2017 at two Referral Centers (University "Luigi Vanvitelli," Naples, Italy, and University "Federico II", Naples, Italy). Inclusion criteria were the following: diagnosis of NF1 according to the recommendations of the National Institutes of Health [23], availability of MRI acquisition, diagnosis of MMS suspected on the MRA data according to the available guidelines [10], and availability of both MRA and DSC-PWI sequences. On the other hand, subjects with the presence of other neurological conditions extending beyond the spectrum of NF1 or with significant artifacts on the neuroradiological images were excluded from this work.

The study was carried out in compliance with the Helsinki Declaration, with all patients that provided a written consent to execution of the imaging exams and for any clinical research purposes. In case of subjects with less than 18 years, the legal guardians provided the required written consent.

\section{MRI data acquisition and processing}

Brain MR scans were all performed on the same $1.5 \mathrm{~T}$ scanner (Gyroscan Intera, Philips Medical System, Best, Netherlands) at a single center. Along with clinical T1-weighted, T2weighted, fluid attenuated inversion recovery (FLAIR), and diffusion weighted imaging (DWI) sequences, MR protocol included a 3D time-of-flight (TOF) MRA for the study of the 
circle of Willis (TR: $22 \mathrm{~ms}$; TE: $7 \mathrm{~ms}$; Flip Angle: $20^{\circ}$; matrix: $304 \times 194$; slice thickness: $1.4 \mathrm{~mm}$ ) and a DSC-PWI sequence (TR: $760 \mathrm{~ms}$; TE: $30 \mathrm{~ms}$; Flip Angle: $40^{\circ}$; matrix: $128 \times 128$; slice thickness: $7 \mathrm{~mm}$; 18 axial slices; 70 volumes; acquisition time: $100 \mathrm{~s}$; temporal resolution: $1.5 \mathrm{~s}$ ). Before the DSC-PWI sequence, a pre-bolus of $1 \mathrm{cc}$ of Gd-DTPA (Gadobutrol, Gadovist ${ }^{\circ}$, Bayer) was administrated to correct T1weighted leakage phenomenon. The DSC-PWI sequence was obtained as follows: 9 dynamic series were acquired before injection, followed by 61 volumes after administration of $0.1 \mathrm{mmol} / \mathrm{Kg}$ of Gd-DTPA and a saline flush of $25 \mathrm{~mL}$ $(2.5 \mathrm{ml} / \mathrm{s})$. DSC-PWI data were then processed offline using Olea Sphere MR perfusion software program, v.3.0 (Olea Medical, La Ciotat, France) with a Bayesian probabilistic method, with automated multiple arterial input function (AIF) selection, to obtain CBF, CBV, MTT, and TTP maps.

\section{SPECT-CBF data acquisition and processing}

Data of $\mathrm{rCBF}$ from SPECT acquisitions were obtained after slow bolus intravenous injection of $51.8 \mathrm{MBq} / \mathrm{Kg}$ of ${ }^{99 \mathrm{~m}} \mathrm{Tc}$ HMPAO (Ceretec $®$, Amersham, UK), according to the procedural guidelines of the European Association of Nuclear Medicine [24]. The radiotracer was injected with the patient lying in supine position with eyes closed in a dimly lit, quiet room. Cerebral activity was recorded in step-and-shoot mode using a dual-head gamma camera equipped with a general purpose, low energy, parallel-hole collimator (E-cam, Siemens Medical Systems). Images were acquired with a $128 \times 128$ matrix for 360 degrees evaluation with a circular orbit. A total of 60 frames were taken at 6-degree intervals of $30 \mathrm{~s}$ for each with a total acquisition time of $30 \mathrm{~min}$. The data were reconstructed with filtered back projection using a Butterworth filter (cutoff 1, order 10), and corrected for attenuation using Chang's algorithm on transaxial images (attenuation factor $0.120 \mathrm{~cm}^{-1}$ ). Coronal and sagittal slices were calculated with the original transaxial images.

\section{Image evaluation}

To evaluate the ability of imaging techniques in the detection of vascular alterations in MMS-NF1 patients, images were analyzed as follows.

All MRI data were evaluated in consensus by two neuroradiologists with more than 8 and 20 years of experience in the field of neuroimaging, blinded to the clinical and nuclear medicine findings, and were asked to establish and report which cerebral artery was more affected by stenosis, also reporting abnormal signal regions on perfusion maps.

Similarly, SPECT images were reviewed in consensus by two experienced nuclear medicine physicians, also with more than 5 and 25 years of experience in the field of neuroimaging, blinded to the clinical diagnosis and MRI findings. Using the cerebellum activity as the reference region for visual inspection, hypoperfused cortical structures were identified.

Finally, to evaluate the correlation between MRI and SPECT data, the two most experienced neuroradiologist and nuclear medicine physicians evaluated in consensus both imaging data.

\section{Results}

Following inclusion and exclusion criteria (Fig. 1), from data available in a cohort of 620 NF1 patients, images of 9 subjects were evaluated in this study $(\mathrm{M} / \mathrm{F}=5 / 4$, mean age: $12.6 \pm$ 4.0 years, age range: $7-21$ years) (Table 1). Furthermore, a SPECT-CBF scan, obtained within 3 months from the MRI, was available in 5 out of 9 patients. Finally, 4 subjects underwent a DSA before a surgical indirect revascularization by encephalo-duro-arterio-myo-synangiosis (EDAMS) procedure.

At the MRI examination, all patients (100.0\%) showed the presence of a vascular narrowing affecting either the ICA or the MCA, while in 3 subjects (33.3\%), an involvement of the posterior circulation was also present. The presence of collaterals was found in 6 patients $(66.7 \%)$, with an increase in CBV values at the level of the subarachnoid spaces of the ipsilateral stenotic artery that was found in $5 / 9$ cases $(55.6 \%)$, due to vessel compensatory dilation phenomena. On the other hand, in only $2 / 9$ cases $(22.2 \%)$, CBV maps showed a significant signal reduction due to chronic hypoperfusion, mainly associated to atrophy and gliosis, while in $3 / 9$ patients $(33.3 \%$ ), no significant asymmetry was found on the CBV maps. Interestingly, in one subject, a complex pattern of cerebral perfusion was found, made of both increased and decreased signal within the same hemisphere. While MRI-CBF was not useful to detect hypoperfused regions, SPECT-CBF was decreased on the same side of the affected vessel in $3 / 5$ patients $(60.0 \%)$. Finally, MTT maps overlapped with the side of the affected cerebrovascular territory in $6 / 9$ subjects $(66.7 \%)$, while TTP maps were even more correlated with the affected side, showing a concordance in $8 / 9$ of the cases $(88.9 \%)$.

When SPECT imaging was performed, concordance of both CBV and TTP measured with MRI was found in $3 / 5$ cases $(60.0 \%)$. Interestingly, among all the DSC-PWI variables, the one providing the highest concordance with SPECT findings was the MTT, showing concordance in all cases $(5 / 5$ patients, $100.0 \%)$.

Two examples of imaging findings are shown in Figs. 2 and 3, while a complete description of all clinical and imaging subjects, with corresponding selected radiological features, is available in Supplementary Materials. 
NF1 patients evaluated between January 2007 and December 2017 $(n=620)$



Fig. 1 The flow diagram showing patient selection

\section{Discussion}

In this case series, we report 9 NF1 patients with vascular involvement undergoing MRI, MRA, and DSC-PWI, describing findings and correlations between these different parameters and reporting two cases of NF1 patients with primitive involvement of the vertebro-basilar system, a finding not described yet in literature, to the best of our knowledge. Furthermore, in 5 subjects, SPECT-CBF data were obtained, and we correlated cerebral perfusion parameters from different imaging techniques, suggesting a possible role of DSC-PWI and, in particular of MTT maps, in the radiological evaluation of these patients.

In NF1 patients, MMS is usually characterized by unilateral stenosis of distal ICA and its branches, whereas progression to bilateral involvement is reported in literature with a variable incidence (10-100\%) [25]. Although both the anterior and the posterior circulations can be affected, the anterior system is usually more frequently and precociously involved [26, 27]. MRI, with particular reference to MRA, is a useful and noninvasive imaging technique that allows for the diagnosis and the follow-up of MMS-NF1 patients [25]. In particular, several authors have examined the accuracy of MRA in the evaluation of vascular involvement in NF1 patients, reporting a sensitivity and specificity for ICA, ACA, and MCA stenosis close to $100 \%$ [28].

In MM patients, dilation of compensatory pial and medullary vessel anastomosis plays a critical role to compensate the decreased brain perfusion pressure [29]. This phenomenon is recognizable on MRI, corresponding to subarachnoid hyperintensity on FLAIR images and leptomeningeal enhancement after gadolinium administration. These findings, known as "ivy sign" and "medullary streaks," respectively $[30,31]$, positively correlate to the CVR reduction and to the onset of ischemic symptoms [32]. In our sample, of the 3 patients with both "ivy sign" and "medullary streaks," 2 subjects showed clinical symptoms referable to cerebral hypoperfusion (patient \#1, with migraine and dizziness, and patient $\# 9$, with history of epilepsy and left hemiparesis), while patient \#2, despite being clinically asymptomatic, already developed an ischemic stroke in the right caudate nucleus, ipsilateral to the ivy sign and to the arterial stenosis.

Cerebral perfusion imaging is crucial in MM patients for the evaluation of hemodynamic variations, before and after surgery $[33,34]$. Different MRI techniques can be used for the study of cerebral perfusion, including ASL and DSC-PWI [35]. Despite ASL represents a less invasive technique compared to DSCPWI, given the absence of contrast administration, it is known to be limited by different factors. In particular, given that the time between labeling in the feeding arteries and the arrival of labeled blood in tissue (arterial transit time, ATT) have a significant effect on the ASL signal and in MMD, the ATT may be prolonged, this could lead to a focal intravascular signal artifacts, with subsequent underestimation of CBF [36]. This limitation, coupled to the notion that NF1 patients are usually affected by CNS neoplasms requiring contrast administration for a proper clinical evaluation, still limits the integration of ALS in clinical practice, indirectly strengthening the role of 


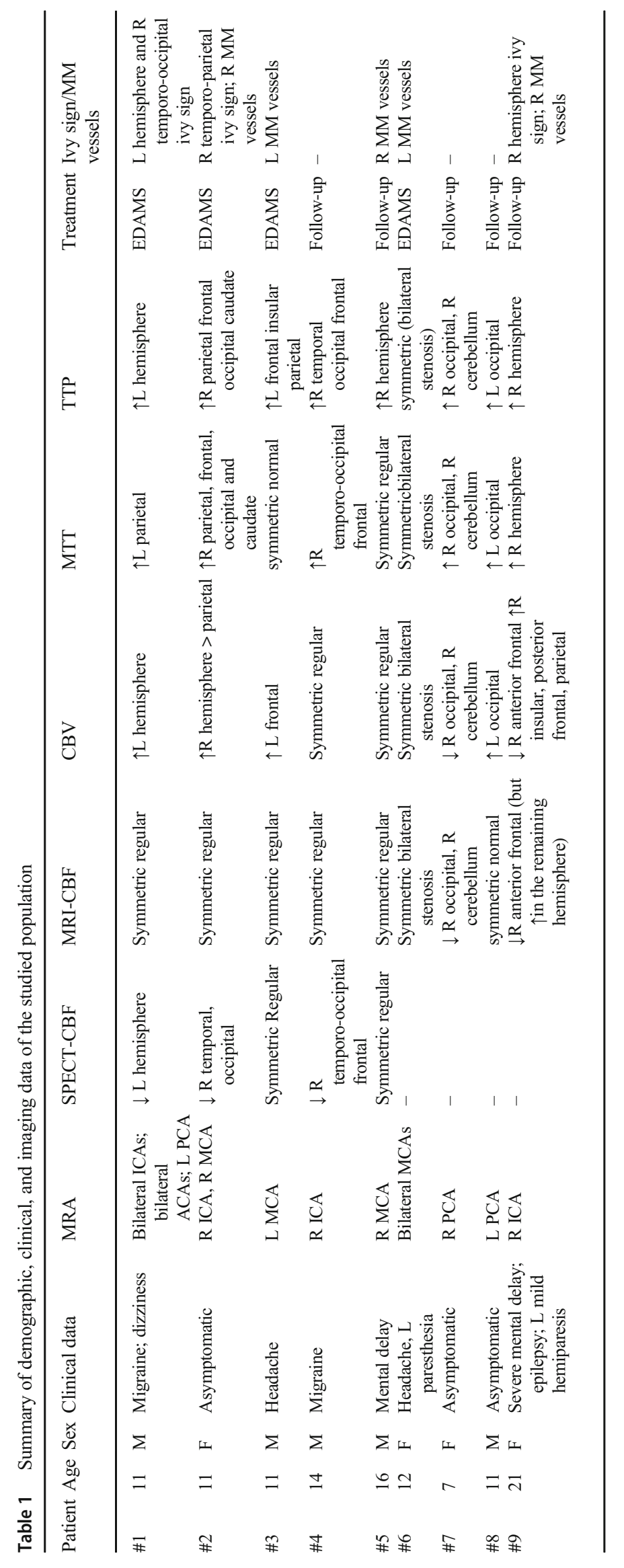




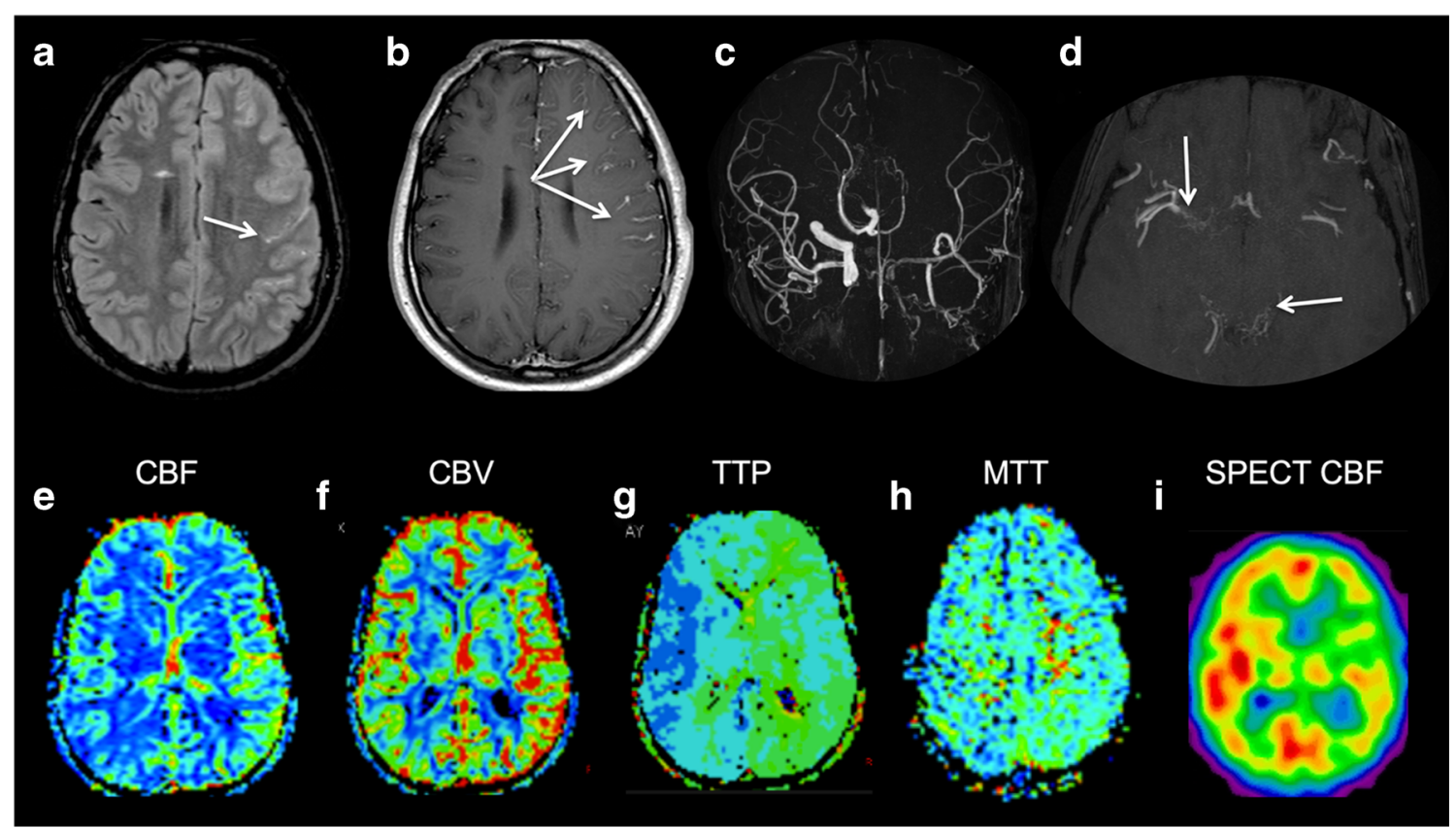

Fig. 2 Imaging findings in patient \#1. FLAIR image (a) shows "ivy sign" in the subarachnoid spaces of the left cerebral hemisphere (arrows) which corresponds to linear enhancement on contrast enhanced T1-weighted sequence (b). MR angiography maximum intensity projection (c) showing left ICA occlusion (related to the presence of a trigeminal neurofibroma infiltrating the cavernous sinus) and multiple MM stenoses on the right distal ICA, A1 segments, and left P3 and P4 segments.
Multiple thin collaterals were observed in the right sylvian fissure and in the quadrigeminal cistern (arrows on d). On DSC-PWI, CBF (e) is symmetric, while CBV (f) and TTP (g) maps show an increase in the left hemisphere. TTP $(\mathbf{g})$, with an increase in MTT $(\mathrm{H})$ at the level of the left parietal lobe. Finally, SPECT demonstrates left cerebral hemisphere hypoperfusion (I)

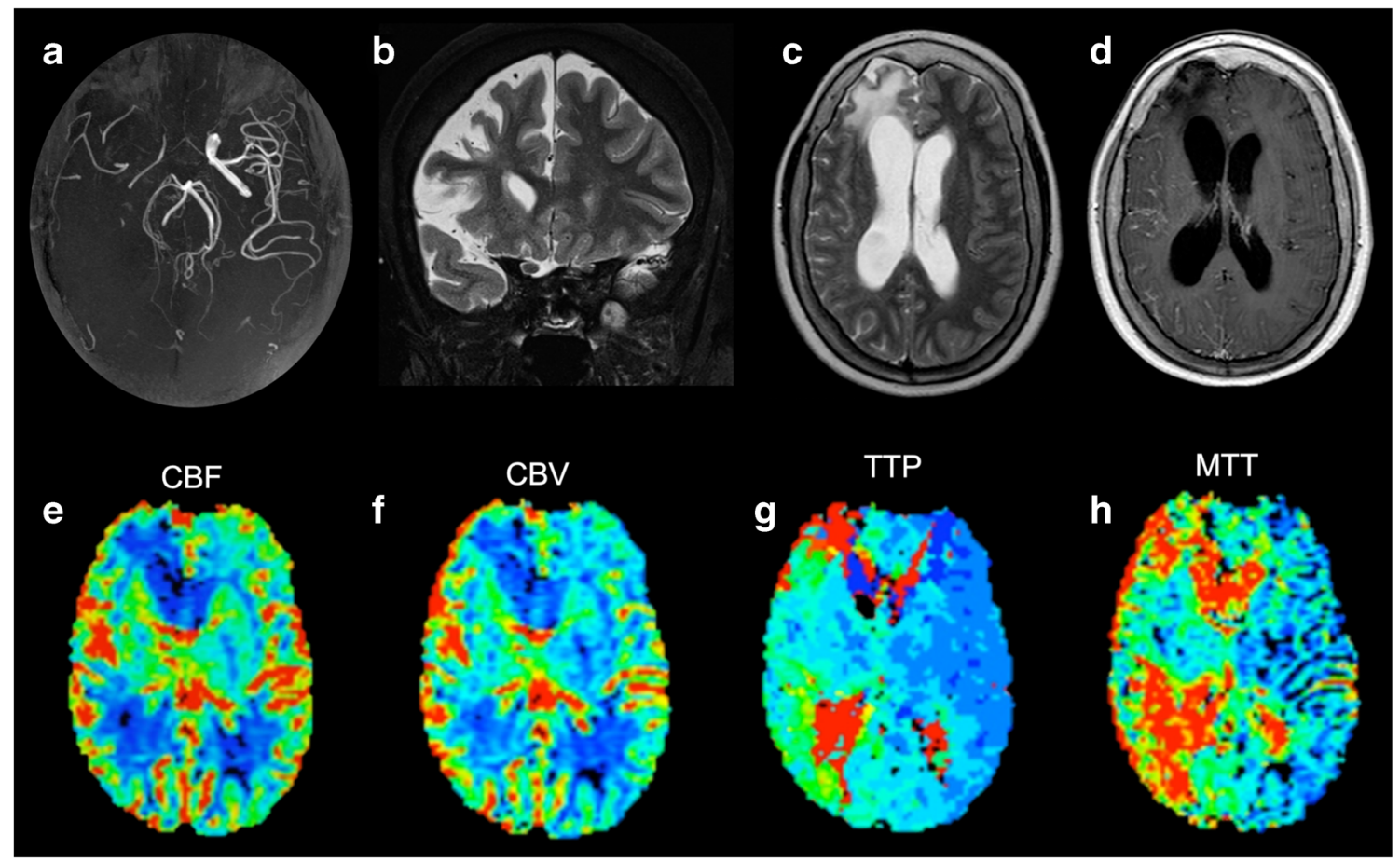

Fig. 3 Imaging findings in patient \#9. Occlusion of the right intracranial ICA is evident on MRA (a). The right frontal lobe appears shrunken and hyperintense on T2-weighted images $(\mathbf{b}-\mathbf{c})$, due to the chronic hypoperfusion. Contrast-enhanced T1-weighted sequence shows ipsilateral leptomeningeal enhancement and MM vessels (d). Reduction of CBF (e) and CBV (f) of the right atrophic frontal lobe was observed, with increase of the whole right hemisphere on TTP (g) and MTT (h) maps. Please note the marked signal increase on the same maps among the remaining right hemisphere 
DSC-PWI as a noninvasive technique to evaluate cerebral perfusion in NF1 patients.

When we evaluated the possible correlation between cerebral perfusion data as measured by SPECT and MRI, we found that MRI-CBF was not useful to detect hypoperfused regions which were conversely identified by SPECT-CBF in 3 of 5 patients $(60 \%)$. This discrepant result is in line with some [37] but not all [38] the previous studies that investigated the relationship between CBF measured by the two different imaging techniques. A possible explanation to this discrepancy could be researched in the different methodological principles underlying CBF measurements. In particular, while SPECT uses a lipophilic tracer to assess $\mathrm{CBF}$ which crosses the brain-blood barrier and permeate into the brain [39], gadolinium used in PWI-MRI is confined in cerebral vessels [40]. Given that the presence of collaterals introduces a wellknown delay and dispersion of the contrast agent bolus in DSC-PWI, their presence introduce an underestimation of CBF (sometimes reported as reaching almost 40\%), making this perfusion map less accurate [41].

In our cases, CBV was increased ipsilaterally to the occluded vessels in 5 patients (55\%), in agreement with the previous reports [42-44]. Arterial dilation, secondary to cerebral hypoperfusion, represents the compensatory mechanism underlying the high signal observable in the cerebral subarachnoid spaces in MM patients. The discrepancy in subjects with preserved CBF, but increased CBV, may be explained by the presence of an early stage cerebral hemodynamic failure [45]. Indeed, when arterial stenosis reduces cerebral perfusion pressure, cerebral arterioles dilate to maintain $\mathrm{CBF}$, leading to an increase in $\mathrm{CBV}$ with a preserved $\mathrm{CBF}$. With further reduction in cerebral perfusion pressure, arterioles reach the maximum dilatation, and therefore, the CBV stop increasing and a decrease in CBF happens [45]. It has been suggested an inverse correlation between MTT and CVR as measured via SPECT with acetazolamide challenge [20], thus representing a noninvasive method to evaluate CVR. In this light, although being limited by a small number of samples, our results partly resemble those available in literature, with MTT maps correlating with SPECT data in all cases. As previously reported [46], these results suggest that MTT is sensitive to cerebral hemodynamic alterations, with its increase that have a significant reliability in the detection of CVR impairment.

Different limitations should be taken into account in this study, mainly related to the small sample size of our population. In particular, although some results are potentially interesting (namely, the correlation between MTT maps and SPECT data), we are aware that these findings are reported in a very small group of patients, that did not even allow for a proper statistical analysis. For this reason, future prospective studies, conducted on larger and heterogeneous populations, are strongly recommended, to further confirm our results.
Nevertheless, our results suggest that DSC-PWI could represent a useful noninvasive technique to evaluate hemodynamic impairment in MMS-NF1 patients. In particular, MTT maps have demonstrated a very good overlap with the $\mathrm{CBF}$ as measured using SPECT, thus encouraging for further studies to confirm a possible role of this perfusion parameter in the radiological evaluation of these patients.

Acknowledgements Open access funding provided by Università degli Studi di Napoli Federico II within the CRUI-CARE Agreement.

\section{Compliance with ethical standards}

Conflict of interest The authors declare that they have no conflict of interest.

Ethical approval All procedures performed in the studies involving human participants were in accordance with the ethical standards of the institutional and/or national research committee and with the 1964 Helsinki Declaration and its later amendments or comparable ethical standards.

Informed consent Informed consent was obtained from all individual participants included in the study. In case of subjects with less than 18 years, the legal guardians provided the required written consent.

Financial disclosures S.C. received fees for speaking from Genzyme and Shire and fees for adv. board from Amicus.

Abbreviations $M R A$, Magnetic resonance angiography; SPECT, Singlephoton emission computed tomography; $C B F$, Cerebral blood flow; $C B V$, Cerebral blood volume; $M T T$, Mean transit time; $T T P$, Time to peak; ICA, Internal carotid artery; $M C A$, Middle cerebral artery; $P C A$, Posterior cerebral artery; $M M$, Moyamoya; EDAMS, Encephalo-duroarterio-myo-synangiosis

Open Access This article is licensed under a Creative Commons Attribution 4.0 International License, which permits use, sharing, adaptation, distribution and reproduction in any medium or format, as long as you give appropriate credit to the original author(s) and the source, provide a link to the Creative Commons licence, and indicate if changes were made. The images or other third party material in this article are included in the article's Creative Commons licence, unless indicated otherwise in a credit line to the material. If material is not included in the article's Creative Commons licence and your intended use is not permitted by statutory regulation or exceeds the permitted use, you will need to obtain permission directly from the copyright holder. To view a copy of this licence, visit http://creativecommons.org/licenses/by/4.0/.

\section{References}

1. D'Amico A, Mazio F, Ugga L, Cuocolo R, Cirillo M, Santoro C, Perrotta S, Melis D, Brunetti A (2018) Medullary unidentified bright objects in neurofibromatosis type 1: a case series. BMC Pediatr 18(1):91. https://doi.org/10.1186/s12887-018-1067-1

2. Santoro C, Di Rocco F, Kossorotoff M, Zerah M, Boddaert N, Calmon R, Vidaud D, Cirillo M, Cinalli G, Mirone G, Giugliano T, Piluso G, D'Amico A, Capra V, Pavanello M, Cama A, Nobili B, Lyonnet S, Perrotta S (2017) Moyamoya syndrome in children with 
neurofibromatosis type 1: Italian-French experience. Am J Med Genet A 173(6):1521-1530. https://doi.org/10.1002/ajmg.a.38212

3. D'Arco F, D'Amico A, Caranci F, Di Paolo N, Melis D, Brunetti A (2014) Cerebrovascular stenosis in neurofibromatosis type 1 and utility of magnetic resonance angiography: our experience and literature review. Radiol Med 119(6):415-421. https://doi.org/10. 1007/s11547-013-0358-8

4. Phi JH, Wang KC, Lee JY, Kim SK (2015) Moyamoya syndrome: a window of moyamoya disease. J Korean Neurosurg Soc 57(6): 408-414. https://doi.org/10.3340/jkns.2015.57.6.408

5. Scott RM, Smith ER (2009) Moyamoya disease and moyamoya syndrome. N Engl J Med 360(12):1226-1237. https://doi.org/10. 1056/NEJMra0804622

6. Feghali J, Xu R, Yang W, Liew JA, Blakeley J, Ahn ES, Tamargo RJ, Huang J (2019) Moyamoya disease versus moyamoya syndrome: comparison of presentation and outcome in 338 hemispheres. J Neurosurg:1-9. https://doi.org/10.3171/2019.6. JNS191099

7. Koc F, Yerdelen D, Koc Z (2008) Neurofibromatosis type 1 association with moyamoya disease. Int J Neurosci 118(8):1157-1163. https://doi.org/10.1080/00207450801898279

8. Rea D, Brandsema JF, Armstrong D, Parkin PC, deVeber G, MacGregor D, Logan WJ, Askalan R (2009) Cerebral arteriopathy in children with neurofibromatosis type 1. Pediatrics 124(3):e476e483. https://doi.org/10.1542/peds.2009-0152

9. Rosser TL, Vezina G, Packer RJ (2005) Cerebrovascular abnormalities in a population of children with neurofibromatosis type 1 . Neurology 64(3):553-555. https://doi.org/10.1212/01.WNL. 0000150544.00016 .69

10. Guidelines for diagnosis and treatment of moyamoya disease (spontaneous occlusion of the circle of Willis) (2012). Neurol Med Chir (Tokyo) 52 (5):245-266. doi:https://doi.org/10.2176/nmc.52.245

11. Mikami T, Sugino T, Ohtaki S, Houkin K, Mikuni N (2013) Diagnosis of moyamoya disease on magnetic resonance imaging: are flow voids in the basal ganglia an essential criterion for definitive diagnosis? J Stroke Cerebrovasc Dis 22(6):862-868. https:// doi.org/10.1016/j.jstrokecerebrovasdis.2012.07.010

12. Derdeyn CP, Zipfel GJ, Zazulia AR, Davis PH, Prabhakaran S, Ivan CS, Aiyagari V, Sagar JR, Hantler N, Shinawi L, Lee JJ, Jafri H, Grubb RL Jr, Miller JP, Dacey RG Jr (2017) Baseline hemodynamic impairment and future stroke risk in adult idiopathic moyamoya phenomenon: results of a prospective natural history study. Stroke 48(4):894-899. https://doi.org/10.1161/STROKEAHA.116.014538

13. Chiu D, Shedden P, Bratina P, Grotta JC (1998) Clinical features of moyamoya disease in the United States. Stroke 29(7):1347-1351. https://doi.org/10.1161/01.str.29.7.1347

14. Kim SK, Cho BK, Phi JH, Lee JY, Chae JH, Kim KJ, Hwang YS, Kim IO, Lee DS, Lee J, Wang KC (2010) Pediatric moyamoya disease: an analysis of 410 consecutive cases. Ann Neurol 68(1): 92-101. https://doi.org/10.1002/ana.21981

15. Liu XJ, Zhang D, Wang S, Zhao YL, Teo M, Wang R, Cao Y, Ye X, Kang S, Zhao JZ (2015) Clinical features and long-term outcomes of moyamoya disease: a single-center experience with 528 cases in China. J Neurosurg 122(2):392-399. https://doi.org/10. 3171/2014.10.JNS132369

16. Zach V, Bezov D, Lipton RB, Ashina S (2010) Headache associated with moyamoya disease: a case story and literature review. J Headache Pain 11(1):79-82. https://doi.org/10.1007/s10194-009-0181-8

17. Shirane R, Fujimura M (2010) Headache in moyamoya disease. In: springer $\mathrm{T}$ (ed) Moyamoya disease update

18. Nariai T, Matsushima Y, Imae S, Tanaka Y, Ishii K, Senda M, Ohno K (2005) Severe haemodynamic stress in selected subtypes of patients with moyamoya disease: a positron emission tomography study. J Neurol Neurosurg Psychiatry 76(5):663-669. https:// doi.org/10.1136/jnnp.2003.025049
19. Ozgur HT, Kent Walsh T, Masaryk A, Seeger JF, Williams W, Krupinski E, Melgar M, Labadie E (2001) Correlation of cerebrovascular reserve as measured by acetazolamide-challenged SPECT with angiographic flow patterns and intra- or extracranial arterial stenosis. AJNR Am J Neuroradiol 22(5):928-936

20. Vagal AS, Leach JL, Fernandez-Ulloa M, Zuccarello M (2009) The acetazolamide challenge: techniques and applications in the evaluation of chronic cerebral ischemia. AJNR Am J Neuroradiol 30(5): 876-884. https://doi.org/10.3174/ajnr.A1538

21. Goetti R, O'Gorman R, Khan N, Kellenberger CJ, Scheer I (2013) Arterial spin labelling MRI for assessment of cerebral perfusion in children with moyamoya disease: comparison with dynamic susceptibility contrast MRI. Neuroradiology 55(5):639-647. https:// doi.org/10.1007/s00234-013-1155-8

22. Hauser TK, Seeger A, Bender B, Klose U, Thurow J, Ernemann U, Tatagiba M, Meyer PT, Khan N, Roder C (2019) Hypercapnic BOLD MRI compared to H2(15)O PET/CT for the hemodynamic evaluation of patients with moyamoya disease. Neuroimage Clin 22:101713. https://doi.org/10.1016/j.nicl.2019.101713

23. Neurofibromatosis. Conference Statement. National Institutes of Health Consensus Development Conference (1988). Arch Neurol 45 (5):575-578

24. Kapucu OL, Nobili F, Varrone A, Booij J, Vander Borght T, Nagren K, Darcourt J, Tatsch K, Van Laere KJ (2009) EANM procedure guideline for brain perfusion SPECT using $99 \mathrm{mTc}$-labelled radiopharmaceuticals, version 2. Eur J Nucl Med Mol Imaging 36(12): 2093-2102. https://doi.org/10.1007/s00259-009-1266-y

25. Vargiami E, Sapountzi E, Samakovitis D, Batzios S, Kyriazi M, Anastasiou A, Zafeiriou DI (2014) Moyamoya syndrome and neurofibromatosis type 1. Ital J Pediatr 40:59. https://doi.org/10.1186/ 1824-7288-40-59

26. Ghosh PS, Rothner AD, Emch TM, Friedman NR, Moodley M (2013) Cerebral vasculopathy in children with neurofibromatosis type 1. J Child Neurol 28(1):95-101. https://doi.org/10.1177/ 0883073812441059

27. Tan C, Duan R, Ye X, Zhang D, Wang R (2016) Posterior circulation moyamoya disease versus primitive vertebral-basilar artery system Moyamoya disease: new classification of moyamoya disease from the perspective of embryology. World Neurosurg 96: 222-229. https://doi.org/10.1016/j.wneu.2016.08.099

28. Sawada T, Yamamoto A, Miki Y, Kikuta K, Okada T, Kanagaki M, Kasahara S, Miyamoto S, Takahashi JC, Fukuyama H, Togashi K (2012) Diagnosis of moyamoya disease using 3-T MRI and MRA: value of cisternal moyamoya vessels. Neuroradiology 54(10): 1089-1097. https://doi.org/10.1007/s00234-012-1020-1

29. Yamamoto A, Okada T, Takahashi JC (2014) Moyamoya disease (spontaneous occlusion of the circle of Willis). In: Saba L, Raz E (eds) Neurovascular Imaging. Springer, New York

30. Maeda M, Tsuchida C (1999) "Ivy sign" on fluid-attenuated inversion-recovery images in childhood moyamoya disease. AJNR Am J Neuroradiol 20(10): 1836-1838

31. Takanashi J, Suzuki H, Barkovich AJ, Sugita K, Saeki N, Kobayashi E, Fujii K, Kohno Y (2003) Medullary streaks: dilated medullary vessels in chronic ischemia in children. Neurology 61(4):583-584. https://doi.org/10.1212/01.wnl.0000076481. $03200.1 \mathrm{f}$

32. Mori N, Mugikura S, Higano S, Kaneta T, Fujimura M, Umetsu A, Murata T, Takahashi S (2009) The leptomeningeal "ivy sign" on fluid-attenuated inversion recovery MR imaging in Moyamoya disease: a sign of decreased cerebral vascular reserve? AJNR Am J Neuroradiol 30(5):930-935. https://doi.org/10.3174/ajnr.A1504

33. Acker G, Fekonja L, Vajkoczy P (2018) Surgical management of moyamoya disease. Stroke 49(2):476-482. https://doi.org/10.1161/ STROKEAHA.117.018563

34. Lee SK, Kim DI, Jeong EK, Kim SY, Kim SH, In YK, Kim DS, Choi JU (2003) Postoperative evaluation of moyamoya disease 
with perfusion-weighted MR imaging: initial experience. AJNR Am J Neuroradiol 24(4):741-747

35. Goetti R, Warnock G, Kuhn FP, Guggenberger R, O'Gorman R, Buck A, Khan N, Scheer I (2014) Quantitative cerebral perfusion imaging in children and young adults with moyamoya disease: comparison of arterial spin-labeling-MRI and H(2)[(15)O]-PET. AJNR Am J Neuroradiol 35(5):1022-1028. https://doi.org/10. 3174/ajnr.A3799

36. Proisy M, Bruneau B, Rozel C, Treguier C, Chouklati K, Riffaud L, Darnault P, Ferre JC (2016) Arterial spin labeling in clinical pediatric imaging. Diagn Interv Imaging 97(2):151-158. https://doi.org/ 10.1016/j.diii.2015.09.001

37. Karonen JO, Vanninen RL, Liu Y, Ostergaard L, Kuikka JT, Nuutinen J, Vanninen EJ, Partanen PL, Vainio PA, Korhonen K, Perkio J, Roivainen R, Sivenius J, Aronen HJ (1999) Combined diffusion and perfusion MRI with correlation to single-photon emission CT in acute ischemic stroke. Ischemic penumbra predicts infarct growth. Stroke 30(8):1583-1590. https://doi.org/10.1161/ 01.str.30.8.1583

38. Nuutinen J, Liu Y, Laakso MP, Karonen JO, Vanninen EJ, Kuikka JT, Aronen HJ, Vanninen RL (2009) Perfusion differences on SPECT and PWI in patients with acute ischemic stroke. Neuroradiology 51(10):687-695. https://doi.org/10.1007/s00234009-0569-9

39. Sogbein OO, Pelletier-Galarneau M, Schindler TH, Wei L, Wells RG, Ruddy TD (2014) New SPECT and PET radiopharmaceuticals for imaging cardiovascular disease. Biomed Res Int 2014:942960 942924. https://doi.org/10.1155/2014/942960

40. Tedeschi E, Caranci F, Giordano F, Angelini V, Cocozza S, Brunetti A (2017) Gadolinium retention in the body: what we know and what we can do. Radiol Med 122(8):589-600. https://doi.org/ 10.1007/s11547-017-0757-3
41. Calamante F, Gadian DG, Connelly A (2000) Delay and dispersion effects in dynamic susceptibility contrast MRI: simulations using singular value decomposition. Magn Reson Med 44(3):466-473. https://doi.org/10.1002/1522-2594(200009)44:3<466::aidmrm18>3.0.co;2-m

42. Calamante F, Ganesan V, Kirkham FJ, Jan W, Chong WK, Gadian DG, Connelly A (2001) MR perfusion imaging in moyamoya syndrome: potential implications for clinical evaluation of occlusive cerebrovascular disease. Stroke 32(12):2810-2816. https://doi.org/ 10.1161/hs1201.099893

43. Tsuchiya K, Inaoka S, Mizutani Y, Hachiya J (1998) Echo-planar perfusion MR of moyamoya disease. AJNR Am J Neuroradiol 19(2):211-216

44. Tzika AA, Robertson RL, Barnes PD, Vajapeyam S, Burrows PE, Treves ST, Scott RM (1997) Childhood moyamoya disease: hemodynamic MRI. Pediatr Radiol 27(9):727-735. https://doi.org/10. $1007 / \mathrm{s} 002470050212$

45. Ito H, Kanno I, Ibaraki M, Hatazawa J, Miura S (2003) Changes in human cerebral blood flow and cerebral blood volume during hypercapnia and hypocapnia measured by positron emission tomography. J Cereb Blood Flow Metab 23(6):665-670. https://doi.org/ 10.1097/01.WCB.0000067721.64998.F5

46. Kawano T, Ohmori Y, Kaku Y, Muta D, Uekawa K, Nakagawa T, Amadatsu T, Kasamo D, Shiraishi S, Kitajima M, Kuratsu J (2016) Prolonged mean transit time detected by dynamic susceptibility contrast magnetic resonance imaging predicts cerebrovascular reserve impairment in patients with moyamoya disease. Cerebrovasc Dis 42(1-2):131-138. https://doi.org/10.1159/000445696

Publisher's note Springer Nature remains neutral with regard to jurisdictional claims in published maps and institutional affiliations. 